Journal of

Molecular Microbiology

and Biotechnology

\title{
Transposon-Mediated Adaptive and Directed Mutations and Their Potential Evolutionary Benefits
}

\author{
Zhongge Zhang Milton H. Saier, Jr. \\ Division of Biological Sciences, Department of Molecular Biology, University of California at San Diego, \\ La Jolla, Calif., USA
}

'If you do not expect to, you will not discover the unexpected.' Heraclitus

\section{Key Words}

Transposon • Insertion sequence $\cdot$ Directed mutation •

Adaptive evolution $\cdot$ Transposition regulation

\begin{abstract}
Transposons, mobile genetic elements that can hop from one chromosomal location to another, are known to be both beneficial and deleterious to the cell that bears them. Their value in accelerating evolutionary adaptation is well recognized. We herein summarize published research dealing with these elements and then move on to review our own research efforts which focus on a small transposon that can induce mutations under the control of host factors in a process that phenotypically and mechanistically conforms to the definition of 'directed mutation'. Directed mutations occur at higher frequencies when they are beneficial, being induced by the stress condition that they relieve. Here, we review evidence for transposon-mediated directed mutation in Escherichia coli. Deletion mutants in the crp gene can not grow on glycerol ( $\left(\mathrm{Glp}^{-}\right)$; however, these cells mutate specifically to efficient glycerol utilization $\left(\mathrm{Glp}^{+}\right)$at rates that are
\end{abstract}

greatly enhanced by the presence of glycerol or the loss of the glycerol repressor (GlpR). These rates are greatly depressed by glucose or by $g l p R$ overexpression. Of the four tandem GlpR-binding sites (01-04) in the control region of the glpFK operon, $\mathrm{O} 4$ (downstream) specifically controls glpFK expression while $\mathrm{O}$ (upstream) controls mutation rate. Mutation is due to insertion of the small transposon IS5 into a specific site just upstream of the glpFK promoter. Mutational control by the glycerol regulon repressor GlpR is independent of the selection and assay procedures, and IS5 insertion into other gene activation sites is unaffected by the presence of glycerol or the loss of GlpR. The results establish the principle of transposon-mediated directed mutation, identify a protein responsible for its regulation, and define essential aspects of the mechanism.

Copyright $\odot 2012$ S. Karger AG, Basel

\section{Introduction}

Transposons are defined as genetic elements that can move or 'hop' from one position in a genome to another. They do so by one of four mechanisms of transposition: (1) conservative (cut and paste or nonreplicative), (2) replicative (the donor and target replicons form a cointegrate without excision, yielding a second copy of the transpo-

\section{KARGER \\ Fax +41613061234 E-Mail karger@karger.ch} www.karger.com

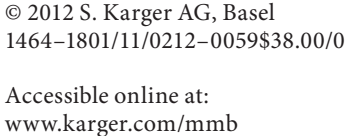

Milton H. Saier, Jr.

Division of Biological Sciences, Department of Molecular Biology

University of California at San Diego

La Jolla, CA 92093-0116 (USA)

Tel. +1 858534 4084,E-Mail msaier@ucsd.edu 
son), (3) excisive (an integrase together with other proteins excises the mobile element, generates a closed circular intermediate and integrates it at the target site, while the DNA remains at the donor site), and (4) retro (an RNA copy of an orf containing the retro-transposon is transcribed, reverse replicated, converted into a cDNA at the insertion site, and the RNA in the DNA site is exchanged for the cDNA). All of these processes, in effect, serve to insert the element into a novel target location. The process uses sitespecific recombination, and is therefore independent of the enzymes of homologous recombination. These genetic elements can be simple (such as a prokaryotic insertion sequence, IS, elements) or complex, bearing genes that allow for other functions such as conjugation and drug resistance in prokaryotes. They may be as small as $0.7 \mathrm{kbp}$ and as large as $500 \mathrm{kbp}$. It is generally agreed that the extraordinary ability of these elements to 'hop' has had a tremendous impact on molecular and organismal evolution.

Transposons can carry useful genes that not only allow hopping within a genome, but also allow for transfer to other organisms via conjugation. Integrons, complex transposons, have two component gene capture and dissemination systems. The first component is an element encoding a site-specific recombinase adjacent to a site of recombination, while the second component consists of gene cassettes that can be inserted and shuffled. These and other mobile elements are perhaps best known because of their contributions to drug resistance in pathogens. Large transposons can also carry genes involved in heavy metal resistance, catabolism and anabolism, vitamin synthesis and nitrogen fixation. They have been found in virtually all types of living organisms. Indeed, transposon-derived DNA can comprise over $50 \%$ of a genome in animals and as much as $90 \%$ in plants [Kazazian, 2004]. Their usefulness as agents of evolutionary change is emphasized by their ability to promote chromosomal rearrangements including deletions, inversions and replicon fusions, events that are central to many evolutionary processes [Schmidt and Anderson, 2006].

In eukaryotes, micro- and macrorearrangements are prevalent, and these can occur for purposes of long or short-term adaptation [Crombach and Hogeweg, 2007]. Their presence can also influence the expression levels of protein-encoding genes [Chung et al., 2007; Marsano et al., 2005]. Moreover, they can be responsive to environmental cues [Schmidt and Anderson, 2006]. It is likely that over evolutionary time, they have promoted the occurrence of adaptive mutations. Mutations are defined as 'adaptive' if they occur randomly but at increased rates under specific stress conditions; mutations are directed if they occur at increased rates specifically under the stress conditions that the mutation relieves.

Retrotransposons not only facilitate genomic rearrangements, they allow meaningful gene reorganization from initially random orders under pressure during evolution [Crombach and Hogeweg, 2007]. They have shaped individual genes as well as genomes [Kazazian, 2004]. Much attention has been devoted to their value as experimental tools for understanding gene function [Grabundzija et al., 2011; Izsvak and Ivics, 2004; Miki et al., 2008].

The documented incidences of transposon-mediated organismal adaptation are numerous. For example, they provide an evolutionarily robust non-telomerase mechanism for the maintenance of telomers in eukaryotes [Pardue and DeBaryshe, 2003]. They influence and are influenced by sexual behavior in some animals [Schaack et al., 2010]. They apparently played crucial roles in the development of antigen-specific immunities [Agrawal et al., 1998; Hiom et al., 1998].

In both prokaryotes and eukaryotes, transposon hopping is controlled not only by environmental conditions, but also by factors that mediate responses to external and internal conditions [Beauregard et al., 2008]. More relevant to the discussion presented here, transposition can be stimulated by nutritional stress in bacteria [Twiss et al., 2005]. In one study, Twiss et al. [2005] described mutations in a gene required for anaerobic fermentative metabolism. These same mutations caused transposon hopping to occur earlier than normal in a developing Escherichia coli colony. Fumarate suppressed this phenotype, providing direct evidence that transposition occurs in response to nutritional stress.

Many proteins have been identified that allow the bacterium to modulate transposition in response to stress conditions. Because transposition can be detrimental as well as beneficial to the organism in which they occur, bacteria have evolved mechanisms that enhance, prevent or regulate transposon movement. Transposons also allow control over target site selection [Martin and Garfinkel, 2003; Scholes et al., 2001]. It is probable that genomes and mobile elements have coevolved to link transpositional dormancy to genome integrity.

In 1943, Luria and Delbrück [1943] reported that bacterial mutations causing phage resistance arose randomly. They suggested that mutations arise independently of selection [Luria and Delbrück, 1943]. Based on this claim and subsequent experiments, it has become a basic principle that mutations occur independently of their phenotypic consequences. The theory of directed mutation, formulated by John Cairns at Harvard University, and de- 
fined as genetic change that is specifically induced by the stress conditions that the mutation relieves, questioned this principle [Cairns and Foster, 1991; Rosenberg, 2001; Wright, 2004].

The topic of directed mutation is controversial, and its potential existence, as defined above, has even been publically denounced [Foster, 1997; Hendrickson et al., 2002; Roth et al., 2006]. In most studies concerning this phenomenon, point mutations were studied [Andersson et al., 1998; Lombardo et al., 2004; Torkelson et al., 1997]. Until recently, the possibility that transposons were capable of causing directed mutation had not been considered. Our work, summarized below, provides the first documented example of directed mutation where the mechanism responsible for the regulation of mutational rate has been largely elucidated [Zhang and Saier, 2009a, b].

\section{Transposon Hopping Can Cause Gene Activation or Inactivation}

Transposons such as insertion sequence 5 (IS5), the most prevalent IS element in E. coli K12, can activate or inactivate genes when inserted in front of or within chromosomal loci [Chandler and Mahillon, 2002; Galas, 1989; Mahillon and Chandler, 1998]. For example, E. coli $n f s A B$ operon products convert nitroaromatic compounds to toxic nitro-anion free radicals via nitroreduction [McCalla, 1979]. In the presence of these compounds in agar plates, resistant colonies appear due to insertion of one of several IS elements, one of which is IS5 [Whiteway et al., 1998]. Activation of expression of the normally cryptic $\beta$ glucoside (bgl) operon in E. coli [Hall, 1998] or the ade gene, encoding adenine deaminase [Petersen et al., 2002], can be accomplished by IS insertion near the promoter. IS5 activates the fucose (Fuc)/propanediol (Ppd) fucAO promoter [Chen et al., 1989] and the flagellar motility flhDC master switch promoter [Barker et al., 2004]. These studies provide the background for our recent research on the E. coli glpFK operon in which IS5-mediated activation occurs in a directed fashion by a well-defined mechanism.

\section{The E. coli glpFK Operon Is Regulated by Crp and GlpR}

The chromosomal $g l p$ regulon of $E$. coli consists of five operons, only one of which, glpFK, is both under Crp (cyclic AMP receptor protein) control and is required for aerobic growth on glycerol [Lin, 1976; Weissenborn et al.,

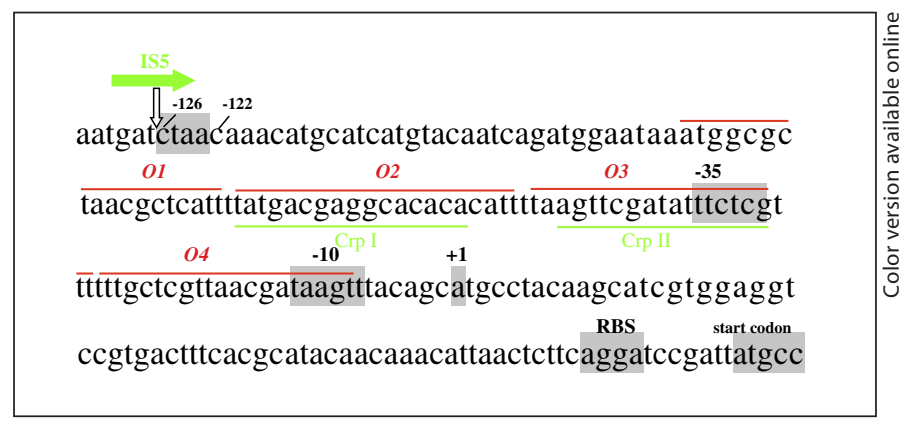

Fig. 1. The E. coli glpFK promoter region. The transcriptional initiation site $(+1)$, the -10 and -35 hexamer that comprise the promoter, the ribosome-binding site (RBS) and the start codon for $g l p F$ translation are shaded. The GlpR-binding sites (O1-O4; lines above the sequence) and Crp-binding sites (CrpI and CrpII; lines under the sequence) are also shown. The location of the IS5 element 37 bp upstream of $\mathrm{O} 1$ in $\operatorname{crp} \mathrm{Glp}^{+}$cells is indicated by the vertical white arrow below the horizontal gray (green in the online version) arrow representing IS5. This arrow shows the orientation of IS5 with the $3^{\prime}$ end of its transposase gene linked to the downstream promoter. The 4-nucleotide IS5 target sequence (CTAA) at -126 to -122 is shaded.

1992]. The $g l p F K$ operon encodes the glycerol facilitator, GlpF, allowing cytoplasmic entry of glycerol at low internal glycerol concentrations, and glycerol kinase, GlpK, phosphorylating cytoplasmic glycerol with ATP, yielding glycerol-3-phosphate and ADP. The glpD gene encodes the aerobic glycerol-3-P dehydrogenase, which oxidizes glycerol-3-P to dihydroxyacetone phosphate [Lin, 1976, 1996]. Both the $g l p F K$ and $g l p D$ operons are subject to negative control by the glp regulon repressor GlpR [Zeng et al., 1996], but $g l p D$ is not regulated by Crp. Glycerol-3phosphate is the inducer of the $g l p$ regulon, binding to GlpR and causing it to dissociate from its operator sites. The fact that $g l p D$ is not regulated by Crp explains why IS5-mediated mutation of $g l p F K$ alone is sufficient to allow crp mutants to grow on glycerol.

The $g l p F K$ operator/promoter region $(\mathrm{PglpFK})$ contains four GlpR-binding sites, O1-O4, and two Crp-binding sites, which overlap $\mathrm{O} 2$ and $\mathrm{O} 3$ (fig. 1). The strong Crp dependency of $g l p F K$ transcription explains the fact that crp mutant cells cannot utilize glycerol.

\section{IS5 Mediates Directed Mutation}

In the absence of Crp, the $g l p F K$ promoter can be activated when IS5 inserts upstream of the promoter [Zhang and Saier, 2009a]. High-level expression of the operon is 


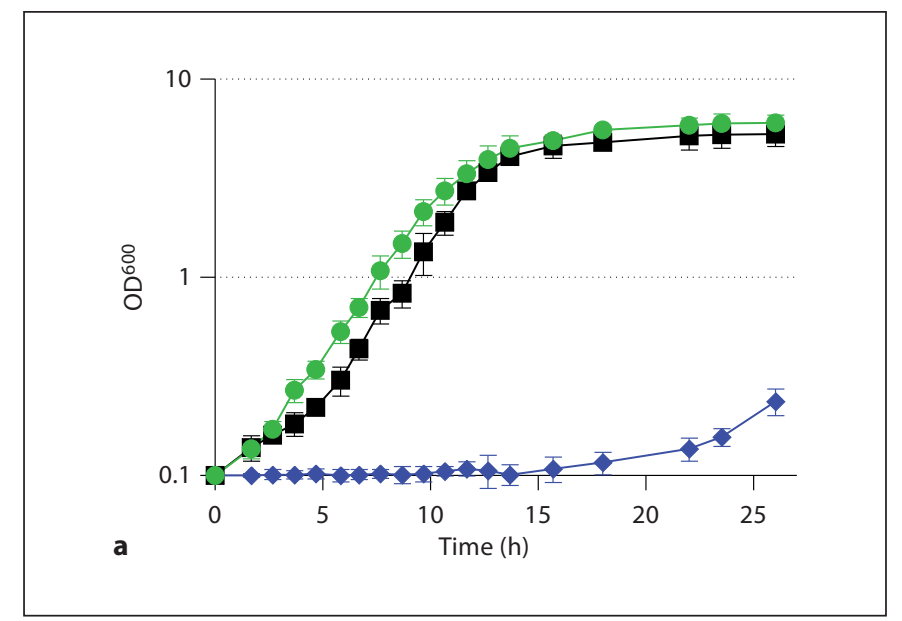

Fig. 2. The appearance of $\mathrm{Glp}^{+}$mutations in a crp genetic background. a Growth of E. coli $w t(\boldsymbol{\square}), \operatorname{crp}(\boldsymbol{\vee})$, and $\operatorname{crp} \mathrm{Glp}^{+}(\boldsymbol{\bullet})$ cells in liquid glycerol minimal medium. $\mathbf{b}$ Growth in the same medium, where the crp mutant cells were plated together with vary-

then nearly constitutive and relies on the DNA phase between the inserted IS5 and the promoter for activation. A short region of $\sim 180$ bps (IB) at the end of IS5 causes activation [Zhang and Saier, 2009b]. This region contains a binding site for the nucleoid protein, IHF, and A-tracts that create a permanent bend [Muramatsu et al., 1988; Saier, 2008]. Moreover, IS5 or IB, when inserted into upstream sites, can activate other Crp-controlled promoters such as the E. coli lactose (lac) promoter [Zhang and Saier, 2009b].

This hopping event appears to represent the first genuinely documented example of directed mutation. $\mathrm{Mu}$ tation is due to IS5 insertion at a single specific site upstream of the $g l p F K$ promoter. A crp deletion strain mutates to $\mathrm{Glp}^{+}$with a $10 \times$ higher frequency when glycerol is present or GlpR is absent. However, overexpression of $g l p R$ depresses the mutation frequency $>100$-fold [Zhang and Saier, 2009a]. Rates of IS5 hopping to sites that activate other promoters are unaffected by glycerol or the loss of GlpR. GlpR therefore serves two distinct biological functions: first, it controls gene expression by binding to the downstream $\mathrm{O} 4$ operator, and second, it controls IS5-dependent $g l p F K$ mutational activation by binding to the upstream $\mathrm{O} 1$ operator, inhibiting transposition to this one site [Zhang and Saier, 2009a]. This is the first example where transposon-mediated directed mutation, involving a DNA-binding protein and occurring by a known molecular mechanism, has been identified.

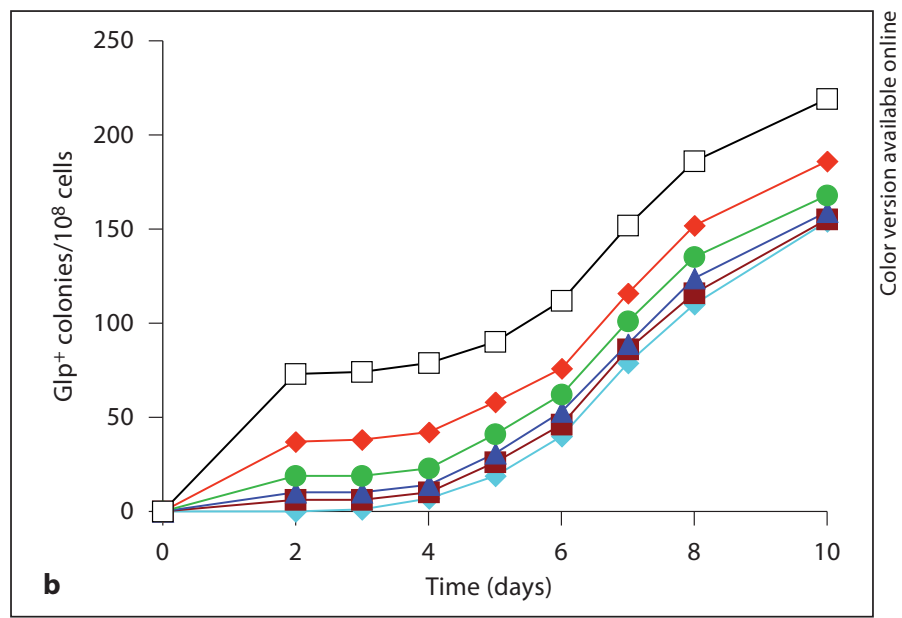

ing numbers of cells from a $\operatorname{crp} \mathrm{Glp}^{+}$strain $(\square, 72 ; \bullet, 38$; $\bullet, 19$; $\boldsymbol{\Delta}, 10 ; \boldsymbol{\square}, 5$, and $\bullet, 0)$. The $\operatorname{crp} \mathrm{Glp}^{+}$cells were mixed with $\operatorname{crp}$ cells $\left(10^{8}\right)$ and then applied onto M9 glycerol agar plates. Six independently isolated $\mathrm{Glp}^{+}$mutant strains behaved the same.

\section{The Activating Hopping Event Is Activated by Glycerol and Prevented by Glucose}

When crp cells of $E$. coli are incubated on solid glycerol minimal medium, $\mathrm{Glp}^{+}$colonies appear at fairly high rates [Zhang and Saier, 2009b]. Independently isolated crp $\mathrm{Glp}^{+}$mutant colonies were purified and examined for growth on glycerol in defined liquid medium. Surprisingly, all such mutants grew at the same rate. A growth curve for one such mutant is shown in figure 2a. The lag phase for the $\operatorname{crp} \mathrm{Glp}^{+}$strain was shorter, and it grew slightly faster than the wild-type E. coli strain. These two strains, the parental $\operatorname{crp} \mathrm{Glp}^{-}$strain and the mutant $\mathrm{crp}$ $\mathrm{Glp}^{+}$strain, oxidized numerous other biological carbon, nitrogen, phosphorous and sulfur sources, at the same rates [Zhang and Saier, 2009b]. Except for the glycerol utilization phenotype, no differences were observed. The mutation that enables crp cells to utilize glycerol apparently affected no other metabolic process.

The relative rates of $\mathrm{Glp}^{+}$mutation were estimated with glycerol, glucitol or glucose as the sole energy source on minimal agar plates. On glycerol plates, colonies first appeared after 3 days, but $w t$ and $c r p \mathrm{Glp}^{+}$cells formed colonies in less than 2 days. New colonies continued to appear at increasing, then decreasing frequencies for several days.

When the same crp cells were plated as before, but a few $\operatorname{crp} \mathrm{Glp}^{+}$cells were mixed with the crp mutant cells before plating, colonies of uniform size appeared before 
$48 \mathrm{~h}$ of incubation. New $\mathrm{Glp}^{+}$mutants arose in parallel to the $\operatorname{crp} \mathrm{Glp}^{+}$cells that appeared on the plates with only $c r p^{-}$cells (fig. 2b). Thus, the $\mathrm{Glp}^{+}$mutants that arose from the crp cells on glycerol minimal medium plates were not present in the cell culture before plating. When glucitol (another polyol crp cells can not utilize) replaced glycerol, the rate of appearance of $\mathrm{Glp}^{+}$mutations was much lower $(>10 \times)$ than that on glycerol plates. On glucose plates, mutation to $\mathrm{Glp}^{+}$could not be detected. The inhibiting effect of glucose proved to be independent of GlpR and appears to be due to a generalized effect on IS5 transposition rather than a target-specific effect on transposition to the $g l p F K$ promoter region [Zhang and Saier, unpubl. obs.].

\section{IS5 Is Responsible for glpFK Operon Activation}

The $g l p F K$ regulatory region from over one hundred $\mathrm{Glp}^{+}$colonies contained a $\sim 1.1-\mathrm{kb}$ insert that proved to be IS5, located at position 126.5 upstream of the $g l p F K$ transcriptional start site. The orientation was always the same with the $3^{\prime}$ end proximally upstream of the promoter (fig. 1). The four-base IS5 targeting sequence, CTAA, was always repeated adjacent to IS5 [Mahillon and Chandler, 1998; Zhang and Saier, 2009b]. No other mutation in addition to the IS5 insertion was detected. Other genetic elements, inserted at the same site, did not activate $g l p F K$ operon expression.

Insertion of a short sequence, either within or downstream of the CTAA target site, essentially abolished the appearance of $\mathrm{Glp}^{+}$mutants. Insertion of the same element upstream of this tetranucleotide sequence reduced the insertion frequency by about $50 \%$. These results showed that (a) the CTAA upstream of PglpFK is essential for IS5 hopping to this site, (b) an appropriate location of the CTAA element upstream of PglpFK is required for glpFK operon activation, and (c) the sequence upstream of the CTAA recognition site maximizes the IS5 insertion rate.

\section{The Glycerol Repressor GIpR Controls the IS5 Hopping Frequency}

Glycerol-3-phosphate binds to and releases the GlpR repressor from its four operators [Lin, 1976; Weissenborn et al., 1992]. We postulated that when GlpR loses affinity for its operators, the DNA configurational change might be transmitted to the IS5 insertion site upstream of PglpFK.
To examine this possibility, $g l p R$ was deleted, and the frequencies of appearance of $\mathrm{Glp}^{+}$mutations in the $\mathrm{crp}$ and the $\operatorname{crp} g l p R$ mutants were measured \pm glycerol. When these two strains were plated without glycerol, $\mathrm{Glp}^{+}$colonies from the double crp $g l p R$ mutant formed on the plates with a 10-fold higher frequency than from the single $\operatorname{crp}$ mutant. In the presence of glycerol, the mutation frequencies were the same. Thus, this rate was the same as observed for the crp glpR double mutant in the absence of glycerol. It was concluded that loss of GlpR has the same affect as the addition of glycerol to the growth medium. GlpR therefore must mediate the response to glycerol [Zhang and Saier, 2009b].

The results summarized above suggest that GlpR binding to its operators blocks the appearance of $\mathrm{Glp}^{+}$ mutations. The $g l p R$ gene was therefore expressed under the control of an arabinose-inducible promoter. When cells expressing $g l p R$ at high levels were examined, the frequencies of appearance of Glp ${ }^{+}$cells decreased well below the background frequency observed for the crp strain under neutral (noninducing, nonrepressing) conditions. Thus, GlpR controls transposition of IS5 to the glpFK upstream target site.

To conform to the definition of 'directed mutation', this effect must be specific to the glpFK promoter. Examination of three other promoters known to be activated by IS5 insertion, the fucAO (Fuc/Ppd utilization) [Chen et al., 1989], flhDC (flagellar master switch) [Barker et al., 2004] and $b g l G F B$ ( $\beta$-glucoside utilization) operons [Reynolds et al., 1981; Schnetz and Rak, 1992], revealed that neither glycerol nor the loss of GlpR influenced IS5 hopping to these sites. Consequently, GlpR specifically influences IS5 transposition to the activating site upstream of the $g l p F K$ operon.

\section{GlpR Binds Different Operators: 04 to Control glpFK Expression, and 01 to Control Glp+ Mutation Frequencies}

DNA footprinting experiments had previously identified four GlpR-binding sites, O1-O4, in the glpFK operon regulatory region [Zeng et al., 1996]. To determine the effects of these operator sites on the two apparent functions of GlpR, the far upstream site (O1) and the far downstream site $(\mathrm{O} 4)$ were mutationally altered, and the effects on $g l p F K$ expression and the IS5 hopping rate were measured [Zhang and Saier, 2009a]. A lacZ reporter gene fusion construct was used to measure $g l p F K$ expression (fig. 3a), and a standard cell counting procedure 


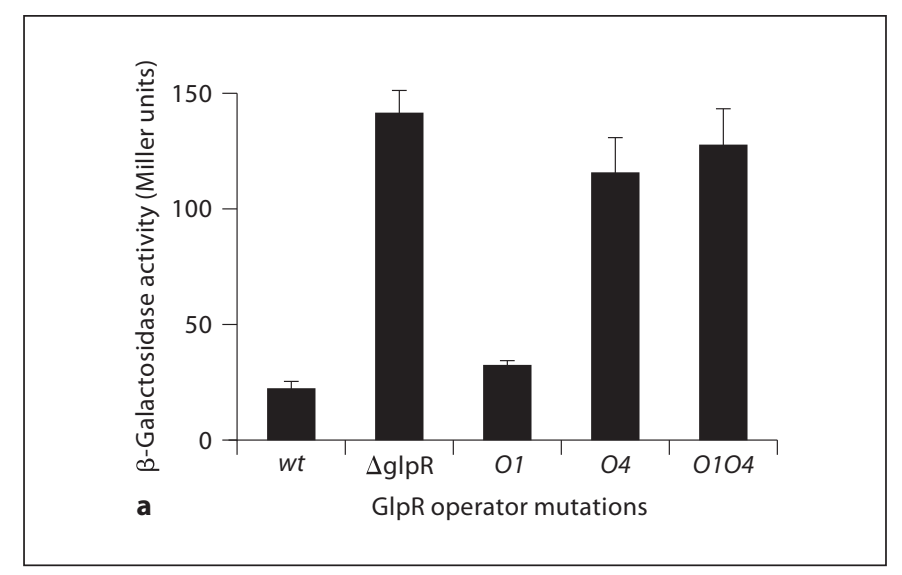

Fig. 3. Effects of the losses of GlpR ( $\Delta$ glpR, deletion of the $g l p R$ gene) and its binding site operators $\mathrm{O} 1$ and $\mathrm{O} 4$ on $g l p F K$ operon expression levels (a) and IS5-mediated mutation rates (b) when grown in liquid LB medium. The frequencies of $\mathrm{Glp}^{+}$mutations relative to the total cell populations were plotted versus time, and the mutation rates were determined from the slopes of the curves. The graphs show the effects of mutations in GlpR and its operators

was used to determine the frequency of mutation to $\mathrm{Glp}^{+}$ (fig. 3b).

Loss of $\mathrm{O} 4$ increased $g l p F K$ operon expression 5 -fold, although mutation of $\mathrm{O} 1$ was nearly without effect (fig. 3a). In contrast, loss of $\mathrm{O} 1$ resulted in a 7 -fold increase in mutation frequency, although loss of $\mathrm{O} 4$ had only a minimal effect (fig. 3b). It seems clear that $O 1$ controls mutation rate while $\mathrm{O} 4$ controls operon expression.

Based on these results, it can be concluded that the two different operators control the two phenomena (operon expression and operon activation), and that consequently, mutation rate is not a function of the $g l p F K$ expression level. GlpR regulates expression and mutation frequencies independently. However, assuming cooperative binding to the four sites, binding to any one of these sites may exert a 'gradient' effect: the closer to $\mathrm{O} 1$ the GlpR-binding site is, the more it will influence mutation rate, while the closer it is to $\mathrm{O} 4$, the more it will influence operon expression. This postulate has yet to be tested.

\section{IS5 Insertion Rates Are Independent of the Selection Procedure}

To test whether control of IS5 hopping by GlpR is independent of the $\mathrm{Glp}^{+}$phenotype, the glpFK operon was replaced with, or was fused to, a chloramphenicol $(\mathrm{Cm})$ resistance (cat) marker. Then, the cat gene was expressed

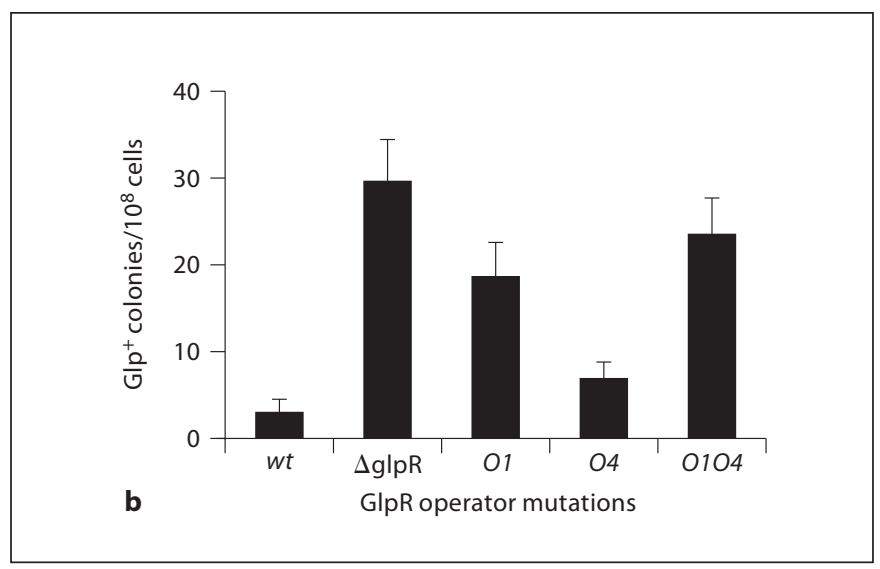

$\mathrm{O} 1$ and $\mathrm{O} 4$ (fig. 1) on PglpFK activity in crp cells grown in LB liquid medium (a), and $\mathrm{Glp}^{+}$mutation frequencies in $c r p$ cells grown on LB solid medium (b). The bars represent standard deviation values for three independently conducted experiments. wt: control with no mutation in either $\mathrm{O} 1$ or 04 ; O1: only operator 1 was mutated; O4: only operator 4 was mutated; O1O4: both operators were mutated.

solely from the $g l p F K$ promoter. $\operatorname{crp}$ and $\operatorname{crp} g l p R$ cells are sensitive to $\mathrm{Cm}$ at $<25 \mu \mathrm{g} / \mathrm{ml}$, but IS5 insertion at the upstream site rendered these cells resistant to $\mathrm{Cm}$ at $>50 \mu \mathrm{g} /$ $\mathrm{ml}$. Using the chromosomal PglpFK-cat construct, IS5 insertion was measured while incubating cells on Luria Bertani broth (LB) agar plates containing $50 \mu \mathrm{g} / \mathrm{ml} \mathrm{Cm}$. Cm-resistant $\left(\mathrm{Cm}^{\mathrm{r}}\right)$ colonies from plates onto which $\mathrm{crp}$ $g l p R$ cells had been plated arose $20 \times$ more frequently for the $\operatorname{crp} g l p R$ cells than for the $\operatorname{crp}$ cells (fig. 4). Moreover, when $g l p R$ was overexpressed, the rate at which $\mathrm{Cm}^{\mathrm{r}}$ colonies appeared decreased over $100 \times$ [Zhang and Saier, 2009b]. Sequencing showed that all independently isolated $\mathrm{Cm}^{\mathrm{r}}$ mutants examined from both the crp and $\mathrm{crp}$ $g l p R$ cells carried IS5 in the usual location and orientation. These observations showed that GlpR inhibits the appearance of $\mathrm{Glp}^{+}$mutations in the absence of glycerol, that IS5 insertion upstream of PglpFK is the cause of the $\mathrm{Glp}^{+}$phenotype, and most importantly, that regulation of mutation rate still occurs when a phenotype unrelated to glycerol metabolism is used to measure the mutation rate.

\section{Glp $^{+}$Mutations Arise in the Absence of Homologous Recombination}

The decreased mutation rate in response to GlpR binding could theoretically have resulted from increased gene dosage accompanying RecA-dependent homologous re- 
combination-dependent partial chromosomal duplications [Petes and Hill, 1988; Roth and Andersson, 2004; Roth et al., 1996]. The dependency of the Glp ${ }^{+}$mutation rate on RecA was therefore examined. When cells were incubated on LB agar plates or on minimal glycerol plates, and the cell population was examined for $\mathrm{Glp}^{+}$and total populations, only a $15 \%$ decrease in mutation rate was observed in crp recA double mutant cells compared to $c r p$ cells. It was concluded that the effect of glycerol and the binding of GlpR to its glpFK operators is not dependent on RecA and therefore is not due to partial chromosomal multiplication.

\section{IS5-Mediated Activation of the glpFK Promoter Is due to DNA Bending and Looping}

IS5 insertion into the unique CTAA target site in front of the $g l p F K$ promoter apparently occurs in $\mathrm{GlpR}^{+}$cells under starvation conditions with high frequency only when glycerol is present and glucose is absent. Of course, the presence of glucose relieves the starvation conditions because $\operatorname{crp}$ mutants can utilize glucose. These starvation conditions plus glycerol are the same conditions under which IS5 insertional mutations are beneficial because their introduction relieves the starvation stress. But what is the mechanism by which IS5 activates the glpFK promoter?

Promoter activation by IS5 is due solely to a short (177bp) region at the 3' end of IS5 [Zhang and Saier, 2009b]. This region proved to be both necessary and sufficient for full promoter activation. It contains two elements: a permanent bend [Ababneh et al., 2008; Tchernaenko et al., 2003, 2004] and a binding site specific for the histone-like nucleoid-binding protein IHF [Saier, 2008]. These two elements account for full glpFK promoter activation [Zhang and Saier, 2009b]. When each was mutated, about $50 \%$ of the activation was lost, and when both were lost, no activation was observed. DNA bending apparently provides the basis for activation by both the permanent bend and IHF. Moreover, phasing proved to be important. In BDNA, a helical turn is about $10.5 \mathrm{bp}$ long. Therefore, if a 10 -bp segment is inserted, the sites before and after the insert should be 'in phase'. However, if a 5 -bp sequence is inserted at the same locus, the two sites should be out of phase by $\sim 180^{\circ}$. When this experiment was concluded, almost all activation was retained for the 10-bp insert, but it was lost when the 5-bp insert was present.

We surmise that the DNA upstream of the glpFK operon interacts with the transcriptional initiation complex

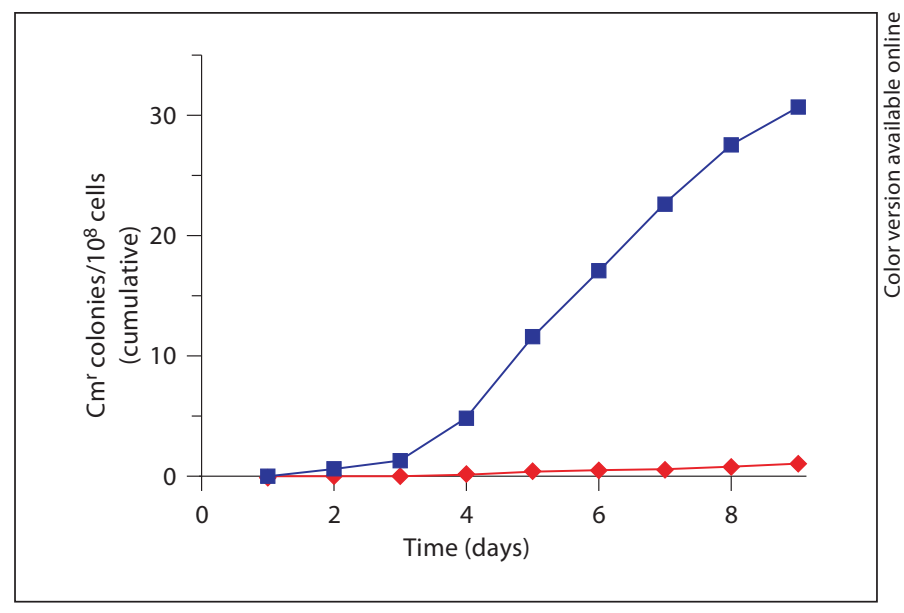

Fig. 4. Effects of GlpR on the frequencies of IS5 insertion upstream of PglpFK when this promoter drives expression of a cat [chlorophenicol resistance $(\mathrm{Cm})]$ gene. The graph reveals the effect of the loss of GlpR on the frequency of IS5 insertion upstream of P $g l p F K$ in $\operatorname{crp}(\checkmark)$ and $\operatorname{crp} g l p R(\boldsymbol{\square})$ genetic backgrounds. GlpR overproduction depressed the rate of mutation below background levels. The results show that control of mutation rate by the binding of GlpR to the DNA is independent of the selection/detection assay.

to activate the promoter that is normally activated by the cyclic AMP-Crp complex in wild-type E. coli cells. Moreover, the lactose (lac) operon proved to be similarly activated by the 177-bp IB fragment in a crp genetic background. These results clearly imply that this newly demonstrated activation mechanism could be applicable to many catabolite-controlled operons, not only in E. coli, but also in all other organisms bearing transposons. Other transposons may have similar capacities to promote activation, although probably by a variety of mechanisms.

\section{Precise Excision of IS5 Can Occur, Rendering Directed Mutation Reversible}

Some transposable elements, but not IS5, had been shown to excise both precisely and imprecisely [Berg, 1977; Botstein and Kleckner, 1977; Khatoon and Bukhari, 1981; Strauch and Beutin, 2006]. The fuc regulon, for Lfucose (Fuc) utilization, consists of two divergent operons, fucPIK and fucAO (fig. 5). The control regions for both operons overlap in the control region separating the two promoters. The fucPIK operon codes for (1) a permease (P) for Fuc uptake, (2) an isomerase (I) that converts Fuc to fuculose, and (3) a kinase (K) that phosphorylates 


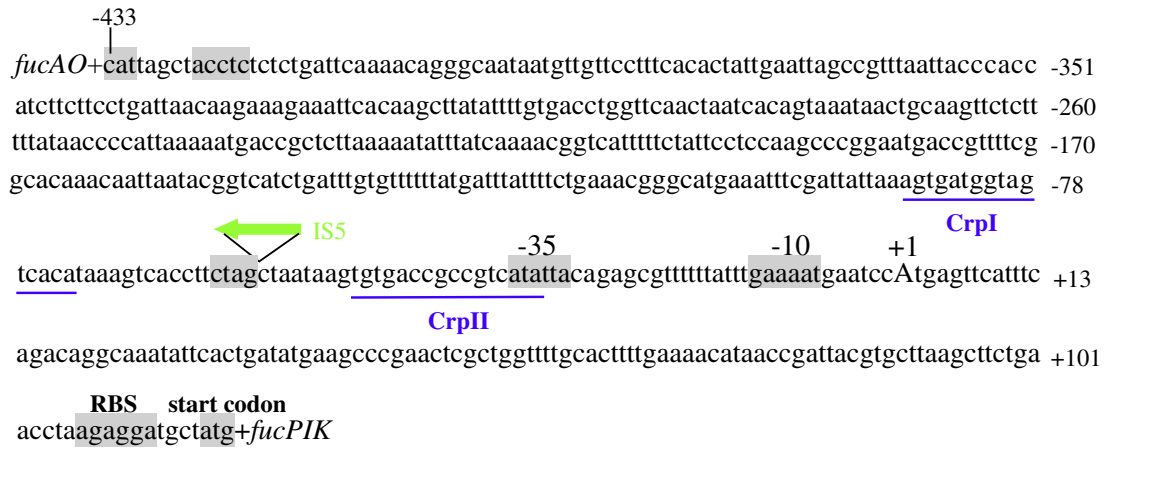

Fig. 5. The interoperonic region between the fucPIK and $f u c A O$ operons. In the $f u c P I K$ promoter region, the transcriptional initiation site $(+1)$ is capitalized. The ctag IS 5 target site, the -10 and -35 hexamers, the ribosome-binding site (RBS) and the ATG start codon for $f u c P$ are shaded. The two putative Crp-binding sites (CrpI and CrpII) are underlined. The gray (green in the online version) backward arrow above the sequence indicates the location of IS5 upstream of the fucPIK operon in $\mathrm{Ppd}^{+} / \mathrm{Fuc}^{-}$cells. The arrow shows

fuculose to fuculose-1-P. In the fucAO operon, fucA codes for an aldolase that cleaves fuculose-1-P to lactaldehyde and dihydroxyacetone-P, while $f u c O$ encodes a reversible oxioreductase that reduces lactaldehyde to Ppd or oxidizes Ppd to lactaldehyde. Under aerobic conditions, lactaldehyde is metabolized to pyruvate that subsequently enters the TCA cycle, but under anaerobic conditions, the product of lactaldehyde reduction is L-1,2-Ppd, and Ppd probably either diffuses out of the cell or is utilized as a carbon source [Chakrabarti et al., 1984]. Expression of both operons relies on Crp as well as the fuc regulon activator FucR. Binding of fuculose-1-P to FucR stimulates transcription of the two divergently transcribed fuc operons [Chakrabarti et al., 1984].

Normally, E. coli cannot utilize Ppd for growth because expression of neither fuc operon is activated appreciably by this carbon source. However, when IS5 inserts into the intergenic region between the two operons (fig. 5), the cells gain the ability to utilize Ppd [Chen et al., 1984, 1989]. The presence of IS5 at its insertion site in the fuc regulon regulatory region causes constitutive expression of the fucAO operon, but blocks expression of the fucPIK operon [Podolny et al., 1999]. IS5 insertion thus allows utilization of $\mathrm{Ppd}\left(\mathrm{Ppd}^{+}\right)$while preventing utilization of Fuc (Fucose ${ }^{-}$[Podolny et al., 1999]. Wild-type cells are $\mathrm{Fuc}^{+} \mathrm{Ppd}^{-}$. However, when IS5 hops into its target site within the regulatory region between the $f u c A O$ and fucPIK operons, fucAO is activated while fucPIK is re- the orientation of IS5 with the defined left end linked to the downstream $f u c A O$ promoter. In the $f u c A O$ promoter region, the putative ribosome-binding site and the start codon for $f u c A$ are shaded. The promoter region for the $f u c A O$ operon is unknown. This operon has been shown to be under positive control by Crp, but the Crpbinding sites have only been identified bioinformatically, not experimentally.

pressed. This results in a change from Fuc $\mathrm{Ppd}^{-}$to $\mathrm{Fuc}^{-}$ $\mathrm{Ppd}^{+}$and provides positive selection for IS5 insertion $\left(\mathrm{Ppd}^{+}\right)$as well as precise IS5 excision ( $\left.\mathrm{Fuc}^{+}\right)$.

Transposons may have evolved in part to effect genetic change by gene activation or inactivation under stressful conditions. If so, it would be likely that under other conditions, such as when Fuc, but not Ppd, is available, the capacity for precise excision of the transposon, restoring the original phenotype, would be beneficial. Without a mechanism to reverse the mutation-induced phenotypic change, transposition could actually be deleterious over long-term evolutionary time.

Using the selection procedures described in the preceding paragraph, precise excision of IS5 from the interoperonic region between $f u c P I K$ and $f u c A O$ could be demonstrated [Zhang et al., 2010]. This was shown, first, by full phenotypic reversion, and second, by DNA sequencing. These experiments therefore allowed establishment of the full reversibility of the IS5 transpositional insertion event. The capacity for precise excision of IS5 increases its potential value for adaptive purposes.

\section{Conclusions and Perspectives}

Directed mutation has been defined as a change in the inherited DNA sequence of an organism that is specifically induced by the stress condition that the mutation re- 
lieves. Although such potential processes involving point mutations have been studied before, the example reviewed here, involving transposon hopping for adaptive purposes, had not been considered. Nor had a directional mutational mechanism been determined [Massey and Buckling, 2002; Reimers et al., 2004; Rosenberg, 2001; Wright et al., 1999]. We here summarized evidence suggesting that IS5 transposition is directed to the glpFK control region, thereby allowing E. coli crp mutants to utilize glycerol.

These mutations are optimally induced by the presence of glycerol under starvation conditions, and GlpR, the $g l p$ regulon-specific protein that regulates mutation frequency, mediates the response to glycerol. Thus, the enhancement mechanism is, in principle, known. GlpR binds to its four operators (O1-O4) overlapping the $g l p F K$ promoter, probably cooperatively. It is displaced from these sites when $\alpha$-glycerol phosphate, derived from exogenous glycerol, binds to it (fig. 1) [Weissenborn et al., 1992]. Thus, GlpR controls both glpFK expression and the IS5 insertional event that produces the $\mathrm{Glp}^{+}$phenotype.

Binding of GlpR to O4, overlapping the -10 region, primarily influences gene expression, while binding to the upstream $\mathrm{O} 1$ primarily influences IS5 transposition into the specific CTAA target site, $126.5 \mathrm{bp}$ upstream of the $g l p F K$ transcriptional start site. This site is only $37 \mathrm{bp}$ upstream of $O 1$ (fig. 1) rendering feasible a conformational change in the DNA, transmitted from $\mathrm{O} 1$ to the CTAA target site. This conformational change could favor IS5 insertion specifically at this site. Alternatively, an inhibitory protein-protein interaction, such as a transposaseGlpR interaction, could account for the results. Either mechanism could explain the activation of transposition to this site by dissociation of GlpR from $\mathrm{Ol}$.

These observations lead to an understanding of how the two functions of GlpR occur independently. The mechanism blocking mutation, dependent on the binding of GlpR to O1, may be direct, promoting interaction of DNA-bound GlpR with the transpososome, or indirect, promoting changes in DNA conformation as noted above. In either case, the physiological consequence is the same: beneficial directed mutation and accelerated adaptation to a changing environment.

Each of the independently isolated $\mathrm{Glp}^{+}$mutants described here contained IS5, always present at the same location and orientation. Only one of the five $g l p$ regulon operons ( $g l p F K$ ) proved to be activated, and activation of only this operon would be expected to be beneficial under the conditions studied. The presence of glycerol or the loss of GlpR affects $g l p F K$ activation, thereby defining the mechanism of IS5-mediated directed mutation. However, these conditions did not influence IS5 transposition into three other expression-activating sites in the E. coli genome, demonstrating specificity. Moreover, there were no other observable phenotypic differences between $\mathrm{Glp}^{+}$ mutants and the parental crp cells. Clearly, mutation to $\mathrm{Glp}^{+}$is directed specifically to the $g l p F K$ operon. These findings make mechanistic and physiological sense, since in all operons shown to be activated by IS5, insertion occurs in the proximity of the promoter that is activated. A chaotic situation would result if such an insertion influenced expression of other more distant genes. Thus, the need for insertion of the transposon near the target operon renders the process specific. The $g l p F K$ system therefore exhibits a phenomenon consistent with the most rigorous definition of true directed mutation. It also reveals potential mechanisms providing the specificity required for conformity to this definition.

Transposon insertion frequencies increase in the presence of specific carbon sources [Hall, 1998, 1999], and various host proteins influence transposition [Chaconas et al., 1996; Chandler and Mahillon, 2002; Galas, 1989; Mahillon and Chandler, 1998]. Many of these proteins are nucleoid-structuring proteins such as H-NS, HU, IHF and Fis [Saier, 2008] or DNA recombination and repair enzymes such as RecG, Dcd and DinD [Swingle et al., 2004; Twiss et al., 2005]. Increased hopping rates have been observed in response to nutritional stress [Twiss et al., 2005]. Moreover, host DNA structuring proteins are involved in assembly of the enzymatic apparatus that comprises the 'transpososome' and mediates target selection [Chalmers et al., 1998; Lavoie and Chaconas, 1993; Surette and Chaconas, 1989; Twiss et al., 2005]. These observations allow recognition of the many different mechanisms that could account for transposon-mediated directed mutation.

The DNA adenine methylase is known to affect transposition of IS5 [Roberts et al., 1985; Yin et al., 1988]. In yeast, multiple host factors control retrotransposon transposition (e.g. Ty1 and Ty3 [Aye et al., 2004; Scholes et al., 2001]). The E. coli RpoS stationary-phase sigma factor has been shown to influence transposition rates as well [Ilves et al., 2001; Moorthy and Mahadevan, 2002; unpubl. obs.]. Based on these facts, it is not surprising that many host proteins influence the overall transposition rates [Chandler and Mahillon, 2002; Galas, 1989; Mahillon and Chandler, 1998; Swingle et al., 2004; Twiss et al., 2005]. The GlpR protein is the latest to have been shown to influence transposition, but in contrast to the others, it does so in a highly specific fashion, determining the transposition frequency to a single site. 


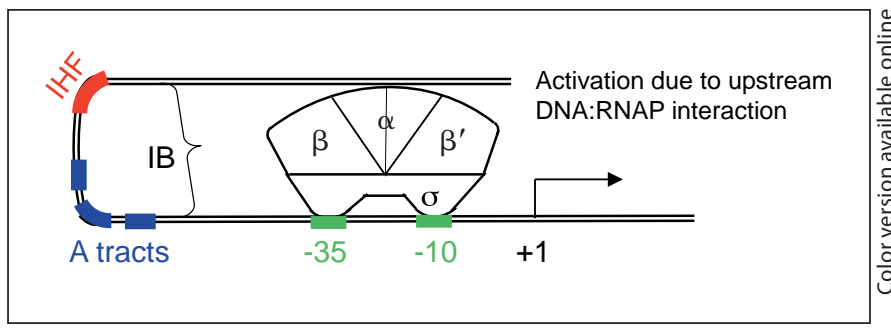

Fig. 6. Model for the activation of $g l p F K$ expression by the upstream IS5 in E. coli K12. IB: IS5 permanent bend/IHF-binding region in the downstream part of IS5; $\alpha, \beta, \beta^{\prime}, \sigma$ : the four dissimilar subunits of $E$. coli RNA polymerase. The -10 and -35 represent the two $\sigma^{70}$-binding sites in the promoter region to which $\sigma^{70}$ binds for open complex formation.

The mechanism recently identified for $g l p F K$ promoter activation provides relief from starvation and therefore could have been selected for through evolutionary time. In a wild-type $\mathrm{Crp}^{+}$background, it could allow rapid utilization of glycerol and abolish diauxie, so that both glucose and glycerol, for example, could be used simultaneously. Glycerol might be used for phospholipid biosynthesis, while glucose could be used as the primary energy source.

Our experimental results suggest that the DNA loop, caused by the permanent bend and IHF binding, could directly contact RNA polymerase to promote transcriptional activation (fig. 6). It appears to be a genuine example of directed mutation. The fact that mutation rate is influenced by the presence of glycerol in a process mediated by the glycerol repressor provides a mechanistic explanation for IS5-mediated directed mutational control (fig. 7). It leads us to propose that GlpR has two functions, one in controlling $g l p$ regulon gene expression as recognized previously, and the other in controlling the conformational state of the upstream DNA so as to influence the rate of IS5 insertion. These observations provide an explanation for the existence of the upstream operator, $\mathrm{Ol}$, in the control region of the $g l p F K$ operon.

Another potential example of directed mutation can be found in the mammalian immune system. Odegard and Schatz [2006] have reviewed evidence for a million-fold increase in mutation rate in humans relative to spontaneous rates of somatic cell mutation, a phenomenon termed somatic hypermutation (SHM). SHM occurs in the variable regions of immunoglobulin (IgG) genes with a rate of up to $10^{-3}$ mutations/bp/cell division. Point mutations can arise in specific tetranucleotide 'hot spots' where the DNA sequence plays an essential role. While our studies have focused on mutations occurring by transposon insertion

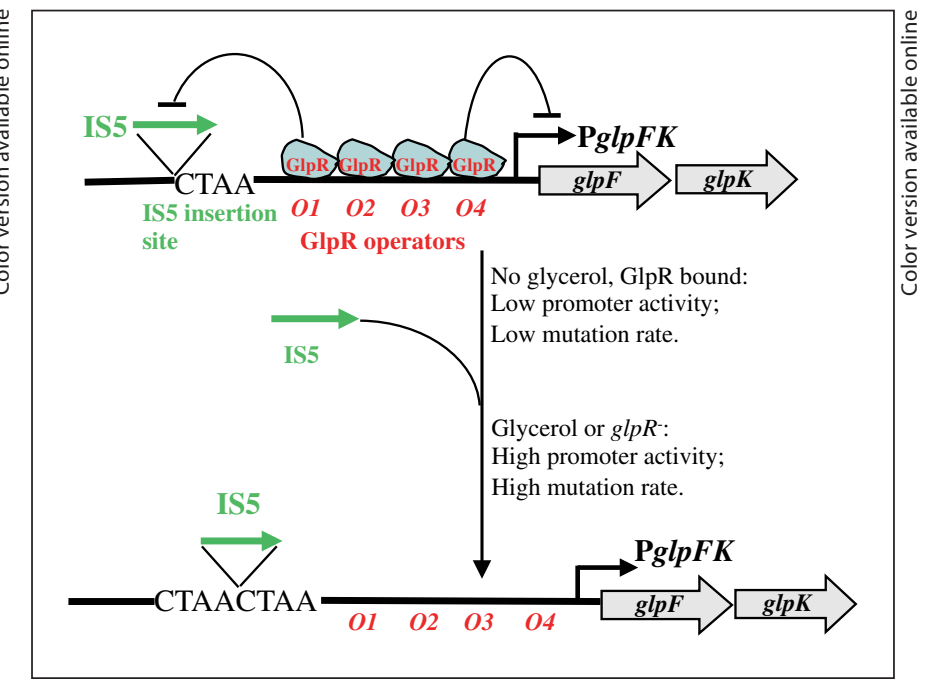

Fig. 7. Schematic diagram illustrating GlpR-mediated control of $g l p F K$ transcription (right) and the rate of IS5 hopping (directed mutation) into the CTAA site upstream of the $g l p F K$ promoter (left). With GlpR bound to its operators (O1-O4), transcription and IS5 hopping both occur at low rates. When GlpR is not bound to its operators, both transcriptional initiation and IS5 hopping increase about 10 -fold. Binding of GlpR to operator O1 blocks IS5 insertion, while binding of GlpR to operator $\mathrm{O} 4$ blocks transcription as indicated.

in E. coli, the IgG system in animals involves the introduction of point mutations. Thus, directed/adaptive point mutations may occur in animal immune systems, even though they have not yet been clearly identified in prokaryotes. It is important to recall, that the inability to demonstrate a phenomenon never proves that it does not exist, but the establishment of one such phenomenon establishes its existence. It is now a question of how significant directed mutation is and has been to organismal evolution.

Reversibility of directed mutation is likely to be more important in some systems than in others. For example, it is essential for the beneficial effects in the bacterial systems that have been reviewed here, and it is only in the bacterial system that reversibility has been demonstrated [Zhang et al., 2010]. It is interesting to note that both processes, IS5 mutation in E. coli, and point mutational 'hot spots' in the variable regions of IgG genes, depend on a specific tetranucleotide sequence.

We find it intuitively appealing that directed mutation could be a property of most or all living cells. It certainly has the potential of benefitting the organism and accelerating genetic adaptation. The evolutionary significance of IS5-mediated control of $g l p F K$ expression in the ab- 
sence of Crp is currently unknown. This transposition event could have evolved to allow simultaneous utilization of two carbon sources (i.e. glucose and glycerol), thus, diminishing the intensity of catabolite repression on $g l p$ gene expression by glucose, and, consequently, abolishing diauxie in wild-type cells. However, it is also possible that the IS5-mediated mechanism of $g l p F K$ expressional control discussed here was an ancient regulatory mechanism that preceded the Crp-cyclic AMP mechanism of control of this same operon. What we have uncovered could be an 'ancient relic' or a vestige of an earlier time. However, we are betting that the same phenomenon will be common as a means of gene control in many organisms. Future studies are likely to provide evidence for the relative importance of directed versus more simple adaptive mutations to organismal evolution. Jumping genes may be as ancient as living organisms themselves. We are just now beginning to appreciate why.

\section{Acknowledgements}

Some figures were reproduced or modified from Zhang and Saier [2009a] or Zhang et al. [2010] with permission. We thank Andrew Dawson and Carl Welliver for the preparation of the manuscript. The research in our laboratory, reviewed here, was supported by NIH grants GM64368 and GM077402.

\section{References}

-Ababneh AM, Ababneh ZQ, Large CC: DNA Atract bending: polarization effects on electrostatic interactions across their minor groove. J Theor Biol 2008;252:742-749.

Agrawal A, Eastman QM, Schatz DG: Transposition mediated by RAG1 and RAG2 and its implications for the evolution of the immune system. Nature 1998;394:744-751.

-Andersson DI, Slechta ES, Roth JR: Evidence that gene amplification underlies adaptive mutability of the bacterial lac operon. Science 1998;282:1133-1135.

- Aye M, Irwin B, Beliakova-Bethell N, Chen E, Garrus J, Sandmeyer S: Host factors that affect Ty3 retrotransposition in Saccharomyces cerevisiae. Genetics 2004;168:1159-1176.

Barker CS, Pruss BM, Matsumura P: Increased motility of Escherichia coli by insertion sequence element integration into the regulatory region of the flhD operon. J Bacteriol 2004;186:7529-7537.

Beauregard A, Curcio MJ, Belfort M: The take and give between retrotransposable elements and their hosts. Annu Rev Genet 2008; 42:587-617.

Berg DE: Insertion and excision of the transposable kanamycin resistance determinant Tn5; in Bukhari AI, Shapiro JA, Adhya SL (eds): DNA Insertion Elements, Plasmids and Episomes. Cold Spring Harbor, Cold Spring Harbor Laboratory, 1977, pp 205-212.

Botstein D, Kleckner N: Translocation and illegitimate recombination by the tetracycline resistance element Tn10; in Bukhari AI, Shapiro JA, Adhya SL (eds): DNA Insertion Elements, Plasmids and Episomes. Cold Spring Harbor, Cold Spring Harbor Laboratory, 1977, pp 185-204.

-Cairns J, Foster PL: Adaptive reversion of a frameshift mutation in Escherichia coli. Genetics 1991;128:695-701.

Chaconas G, Lavoie BD, Watson MA: DNA transposition: jumping gene machine, some assembly required. Curr Biol 1996;6:817-820.
Chakrabarti T, Chen YM, Lin EC: Clustering of genes for L-fucose dissimilation by Escherichia coli. J Bacteriol 1984;157:984-986.

Chalmers R, Guhathakurta A, Benjamin H, Kleckner N: IHF modulation of Tn10 transposition: sensory transduction of supercoiling status via a proposed protein/DNA molecular spring. Cell 1998;93:897-908.

Chandler M, Mahillon J: Insertion sequences revised; in Craig NL, Craig R, Gellert M, Lam bowitz AM (eds): Mobile DNA II. Washington, ASM Press, 2002, pp 305-366.

Chen YM, Chakrabarti T, Lin EC: Constitutive activation of L-fucose genes by an unlinked mutation in Escherichia coli. J Bacteriol 1984 159:725-729.

Chen YM, Lu Z, Lin EC: Constitutive activation of the fucAO operon and silencing of the divergently transcribed fucPIK operon by an IS5 element in Escherichia coli mutants selected for growth on L-1,2-propanediol. J Bacteriol 1989;171:6097-6105.

Chung $\mathrm{H}$, Bogwitz MR, McCart C, Andrianopoulos A, French-Constant RH, Batterham P, Daborn PJ: Cis-regulatory elements in the Accord retrotransposon result in tissue-specific expression of the Drosophila melanogaster insecticide resistance gene $C y$ p6g1. Genetics 2007;175:1071-1077.

Crombach A, Hogeweg P: Chromosome rearrangements and the evolution of genome structuring and adaptability. Mol Biol Evol 2007;24:1130-1139.

Foster PL: Nonadaptive mutations occur on the F' episome during adaptive mutation conditions in Escherichia coli. J Bacteriol 1997;179: 1550-1554.

Galas DJ: Bacterial insertion sequences; in Berg D, Howe M (eds): Mobile DNA. Washington, American Society for Microbiology, 1989, pp 109-162.
Grabundzija I, Izsvak Z, Ivics Z: Insertional engineering of chromosomes with Sleeping Beauty transposition: an overview. Methods Mol Biol 2011;738:69-85.

Hall BG: Activation of the $b g l$ operon by adaptive mutation. Mol Biol Evol 1998;15:1-5.

Hall BG: Spectra of spontaneous growth-dependent and adaptive mutations at ebgR. J Bacteriol 1999;181:1149-1155.

Hendrickson H, Slechta ES, Bergthorsson U, Andersson DI, Roth JR: Amplification-mutagenesis: evidence that 'directed' adaptive mutation and general hypermutability result from growth with a selected gene amplification. Proc Natl Acad Sci USA 2002;99:21642169.

Hiom K, Melek M, Gellert M: DNA transposition by the RAG1 and RAG2 proteins: a possible source of oncogenic translocations. Cell 1998;94:463-470.

- Ilves H, Horak R, Kivisaar M: Involvement of sigmaS in starvation-induced transposition of Pseudomonas putida transposon Tn4652. J Bacteriol 2001;183:5445-5448.

Izsvak Z, Ivics Z: Sleeping beauty transposition: biology and applications for molecular therapy. Mol Ther 2004;9:147-156.

Kazazian HH Jr: Mobile elements: drivers of genome evolution. Science 2004;303:16261632.

Khatoon H, Bukhari AI: DNA rearrangements associated with reversion of bacteriophage $\mathrm{Mu}-$ induced mutations. Genetics 1981;98:1-24.

Lavoie BD, Chaconas G: Site-specific HU binding in the $\mathrm{Mu}$ transpososome: conversion of a sequence-independent DNA-binding protein into a chemical nuclease. Genes Dev 1993; 7:2510-2519.

Lin EC: Glycerol dissimilation and its regulation in bacteria. Annu Rev Microbiol 1976;30: 535-578.

Lin EC: Dissimilatory pathways for sugars, polyols, and carboxylates. Escherichia coli and Salmonella: Cell Mol Biol 1996:307-342. 
Lombardo MJ, Aponyi I, Rosenberg SM: General stress response regulator RpoS in adaptive mutation and amplification in Escherichia coli. Genetics 2004;166:669-680.

-Luria SE, Delbrück M: Mutations of bacteria from virus sensitivity to virus resistance. $\mathrm{Ge}$ netics 1943;28:491-511.

Mahillon J, Chandler M: Insertion sequences. Microbiol Mol Biol Rev 1998;62:725-774.

-Marsano RM, Caizzi R, Moschetti R, Junakovic $\mathrm{N}$ : Evidence for a functional interaction between the Baril transposable element and the cytochrome $\mathrm{P} 450$ cyp12a4 gene in Drosophila melanogaster. Gene 2005;357:122-128.

-Martin SL, Garfinkel DJ: Survival strategies for transposons and genomes. Genome Biology 2003;4:313.

-Massey RC, Buckling A: Environmental regulation of mutation rates at specific sites. Trends Microbiol 2002;10:580-584.

McCalla DR: Mechanism of Action of Antibacterial Agents. Berlin, Springer, 1979.

Miki T, Yamamoto Y, Matsuda H: A novel, simple, high-throughput method for isolation of genome-wide transposon insertion mutants of Escherichia coli K-12. Methods Mol Biol 2008;416:195-204.

Moorthy S, Mahadevan S: Differential spectrum of mutations that activate the Escherichia coli bgl operon in an rpoS genetic background. J Bacteriol 2002;184:4033-4038.

-Muramatsu S, Kato M, Kohara Y, Mizuno T: Insertion sequence IS5 contains a sharply curved DNA structure at its terminus. Mol Gen Genet 1988;214:433-438.

-Odegard VA, Schatz DG: Targeting of somatic hypermutation. Nat Rev Immunol 2006;6: 573-583.

- Pardue ML, DeBaryshe PG: Retrotransposons provide an evolutionarily robust non-telomerase mechanism to maintain telomeres. Annu Rev Genet 2003;37:485-511.

Petersen C, Moller LB, Valentin-Hansen P: The cryptic adenine deaminase gene of Escherichia coli. Silencing by the nucleoid-associated DNA-binding protein, H-NS, and activation by insertion elements. J Biol Chem 2002;277:31373-31380.

Petes TD, Hill CW: Recombination between repeated genes in microorganisms. Annu Rev Genet 1988;22:147-168.

- Podolny V, Lin EC, Hochschild A: A cyclic AMP receptor protein mutant that constitutively activates an Escherichia coli promoter disrupted by an IS5 insertion. J Bacteriol 1999; 181:7457-7463.
Reimers JM, Schmidt KH, Longacre A, Reschke DK, Wright BE: Increased transcription rates correlate with increased reversion rates in leuB and $\operatorname{argH}$ Escherichia coli auxotrophs. Microbiology 2004;150:1457-1466.

Reynolds AE, Felton J, Wright A: Insertion of DNA activates the cryptic $b g l$ operon in $E$. coli K12. Nature 1981;293:625-629.

Roberts D, Hoopes BC, McClure WR, Kleckner $\mathrm{N}$ : IS10 transposition is regulated by DNA adenine methylation. Cell 1985;43:117130.

Rosenberg SM: Evolving responsively: adaptive mutation. Nat Rev Genet 2001;2:504-515.

Roth JR, Andersson DI: Amplification-mutagenesis - how growth under selection contributes to the origin of genetic diversity and explains the phenomenon of adaptive mutation. Res Microbiol 2004;155:342-351.

Roth JR, Benson N, Galitski T, Haack K, Lawrence J, Miesel L: Rearrangements of the bacterial chromosome: formation and application; in Neidhardt F, Ingraham J, Low K, Magasanik B, Schaechter M, Umbarger $\mathrm{H}$ (eds): Escherichia coli and Salmonella typhimurium: Cellular and Molecular Biology. Washington, ASM Press, 1996, pp 22562276.

Roth JR, Kugelberg E, Reams AB, Kofoid E, Andersson DI: Origin of mutations under selection: the adaptive mutation controversy. Annu Rev Microbiol 2006;60:477-501.

Saier MH Jr: The bacterial chromosome. Crit Rev Biochem Mol Biol 2008;43:89-134.

$\checkmark$ Schaack S, Choi E, Lynch M, Pritham EJ: DNA transposons and the role of recombination in mutation accumulation in Daphnia pulex. Genome Biol 2010;11:R46.

Schmidt AL, Anderson LM: Repetitive DNA elements as mediators of genomic change in response to environmental cues. Biol Rev Camb Philos Soc 2006;81:531-543.

Schnetz K, Rak B: IS5: a mobile enhancer of transcription in Escherichia coli. Proc Natl Acad Sci USA 1992;89:1244-1248.

-Scholes DT, Banerjee M, Bowen B, Curcio MJ: Multiple regulators of Tyl transposition in Saccharomyces cerevisiae have conserved roles in genome maintenance. Genetics 2001; 159:1449-1465.

Strauch E, Beutin L: Imprecise excision of insertion element IS5 from the fliC gene contributes to flagellar diversity in Escherichia coli. FEMS Microbiol Lett 2006;256:195-202.

Surette MG, Chaconas G: A protein factor which reduces the negative supercoiling requirement in the Mu DNA strand transfer reaction is Escherichia coli integration host factor. J Biol Chem 1989;264:3028-3034.

- Swingle B, O'Carroll M, Haniford D, Derbyshire KM: The effect of host-encoded nucleoid proteins on transposition: H-NS influences targeting of both IS903 and Tn10. Mol Microbiol 2004;52:1055-1067.
Tchernaenko V, Halvorson HR, Lutter LC: Topological measurement of an A-tract bend angle: variation of duplex winding. J Mol Biol 2003;326:751-760.

Tchernaenko V, Halvorson HR, Lutter LC: Topological measurement of an A-tract bend angle: effect of magnesium. J Mol Biol 2004; 341:55-63.

Torkelson J, Harris RS, Lombardo MJ, Nagendran J, Thulin C, Rosenberg SM: Genomewide hypermutation in a subpopulation of stationary-phase cells underlies recombination-dependent adaptive mutation. EMBO J 1997; 16:3303-3311.

Twiss E, Coros AM, Tavakoli NP, Derbyshire KM: Transposition is modulated by a diverse set of host factors in Escherichia coli and is stimulated by nutritional stress. Mol Microbiol 2005;57:1593-1607.

-Weissenborn DL, Wittekindt N, Larson TJ: Structure and regulation of the $g l p F K$ operon encoding glycerol diffusion facilitator and glycerol kinase of Escherichia coli K-12. J Biol Chem 1992;267:6122-6131.

Whiteway J, Koziarz P, Veall J, Sandhu N, Kumar P, Hoecher B, Lambert IB: Oxygen-insensitive nitroreductases: analysis of the roles of $n f_{s} A$ and $n f_{s} B$ in development of resistance to 5-nitrofuran derivatives in Escherichia coli. J Bacteriol 1998;180:5529-5539.

Wright BE: Stress-directed adaptive mutations and evolution. Mol Microbiol 2004;52:643650 .

$\checkmark$ Wright BE, Longacre A, Reimers JM: Hypermutation in derepressed operons of Escherichia coli K12. Proc Natl Acad Sci USA 1999;96: 5089-5094.

Yin JC, Krebs MP, Reznikoff WS: Effect of Dam methylation on Tn 5 transposition. J Mol Biol 1988;199:35-45.

Zeng G, Ye S, Larson TJ: Repressor for the snglycerol 3-phosphate regulon of Escherichia coli K-12: primary structure and identification of the DNA-binding domain. J Bacteriol 1996;178:7080-7089.

Zhang Z, Saier MH Jr: A mechanism of transposon-mediated directed mutation. Mol Microbiol 2009a;74:29-43.

Zhang Z, Saier MH Jr: A novel mechanism of transposon-mediated gene activation. PLoS Genet 2009b;5:e1000689.

Z Zhang Z, Yen MR, Saier MH Jr: Precise excision of IS5 from the intergenic region between the fucPIK and the fucAO operons and mutational control of $f u c P I K$ operon expression in Escherichia coli. J Bacteriol 2010;192:20132019. 